\title{
La formation des enseignants à Genève
}

Université de Genève

Daniel Peraya

Université de Genève

\section{Chronique internationale}

\section{Les particularités du contexte suisse}

Depuis sa naissance en 1848, la Confédération helvétique est une structure décentralisée, fondée en premier lieu sur ses communes et ses cantons, le pouvoir central ne s'exerçant en son sein que dans des domaines limités et bien précis dont la prise en charge nationale paraît indispensable pour ériger un État-nation. Ainsi, l'instruction publique qui émerge à cette époque est d'emblée clairement placée sous la responsabilité de chaque canton et par conséquent développée selon des logiques et dans des systèmes différents en fonction de spécificités politiques, culturelles, linguistiques ou encore religieuses. Cette remarquable hétérogénéité des situations, bien différente de l'organisation centralisée qui s'observe dans des pays voisins, ne produit pas des enseignements radicalement différents, mais relève d'une organisation spécifique qui crée parfois des difficultés pour des familles se déplaçant d'un canton à l'autre. Elle est par ailleurs nuancée par des processus d'harmonisation récents qui touchent notamment les calendriers scolaires, les objectifs de formation ${ }^{1}$ et les plans d'études de la scolarité obligatoire au sein de chaque zone linguistique ${ }^{2}$, ainsi que la reconnaissance de l'examen de maturité marquant pour les élèves la fin des études gymnasiales, soit la fin de l'enseignement secondaire post obligatoire, appelé aussi le secondaire $\mathrm{II}^{3}$.

Ces disparités régionales expliquent la grande diversité des situations cantonales en ce qui concerne la formation des enseignants. Les éléments de différenciation portent ici à la fois sur la distinction ou non de la formation académique 
et de la formation pédagogique, sur les lieux de cette formation - hautes écoles pédagogiques ou universités -, sur les manières de différencier ou non les trois niveaux d'enseignement (primaire, secondaire I et secondaire II), ou encore sur les façons de relier la formation théorique et la formation pratique en fonction des liens avec le terrain (stages). Ajoutons toutefois que, ces dernières années, la mise en place d'un processus d'harmonisation nationale de l'examen de maturité pour qu'il donne bien accès à toutes les universités mène également à une harmonisation des contenus, ainsi qu'à des exigences précises, en termes de crédits ECTS, de la formation professionnelle initiale des enseignants à qui cet examen de maturité est confié. La Conférence suisse de directeurs cantonaux de l'instruction publique (CDIP), une structure qui émane bien des cantons et non pas de la Confédération, est ainsi l'organe responsable de la reconnaissance des diplômes des enseignants comme de ceux des élèves.

\section{La formation des enseignants : en Suisse et dans le canton de Genève}

À l'échelle nationale, un processus de réforme de la formation des enseignants s'est engagé depuis les années 1990, marqué notamment par des intentions d'harmonisation et de tertiarisation (Criblez,2010; Schneuwly, Heimberg et Villemin, 2012), associant aussi bien l'intégration d'un accès à la recherche que la centralité d'une formation pratique. Longues et complexes, les procédures de reconnaissance des formations d'enseignants qui sont en cours ne mènent pas pour autant à leur uniformisation. Des spécificités significatives demeurent, selon par exemple l'importance plus ou moins grande qui est donnée à la distinction de l'enseignement gymnasial par rapport aux autres (beaucoup plus forte dans les cantons alémaniques), entre des cantons universitaires et d'autres qui ne le sont pas, en fonction des regroupements éventuels de plusieurs cantons pour l'organisation de la formation des enseignants (comme Neuchâtel, le Jura et la partie francophone du canton de Berne), etc.

Il ne nous est pas possible de décrire et d'expliquer ici de manière exhaustive tout ce qui distingue les cantons suisses en matière de formation des enseignants. Une bonne partie des cantons assurent cette formation dans des hautes écoles pédagogiques, des hautes écoles qui ne sont pas universitaires. Mais un canton comme Fribourg sépare la formation au primaire, assurée par une haute école, et la formation pour le secondaire, développée dans son université. Nous allons donc nous concentrer ici sur deux enjeux clés de cette formation à partir du cas genevois.

Dans le canton de Genève, la formation des enseignants est universitaire aussi bien pour l'enseignement primaire que pour le secondaire. Une autre spécificité genevoise concerne la stricte identité de statut et de formation des enseignants des deux niveaux de l'enseignement secondaire, y compris pour les écoles gymnasiales, ce qui implique une double conformité aux exigences de la CDIP qui mettent respectivement l'accent sur les contenus de sciences de l'éducation pour le secondaire I et sur les contenus académiques pour le niveau gymnasial. Enfin, autre élément d'originalité pour les deux niveaux du secondaire, la formation professionnalisante des enseignants, marquée par une forte articulation entre des éléments théoriques et une expérience pratique de terrain, comprend un stage en responsabilité d'une année à mi-temps au cours duquel l'étudiant est pleinement en charge des enseignements qui lui sont attribués, lesquels sont observés et analysés dans le cadre de la formation (Schneuwly et al., 2012). 
Nous allons donc essentiellement nous concentrer ici sur deux aspects, aussi bien pour le primaire que pour le secondaire :

- les liens entre les savoirs de référence et leur enseignement, autour de la transposition didactique;

- l'articulation entre les savoirs théoriques et la formation pratique sur le terrain.

\section{Des savoirs de référence à leur enseignement et apprentissage}

La formation des enseignants peut se présenter comme étant intégrée, c'est généralement le cas pour les enseignants du primaire, ou consécutive à une formation universitaire dans une ou plusieurs disciplines enseignables, ce qui s'observe pour l'enseignement secondaire. Elle est constituée en particulier de contenus didactiques et de contenus de sciences de l'éducation, la dimension didactique se trouvant forcément au centre de l'articulation entre théorie et pratique de terrain pour permettre l'instillation d'une dimension réflexive au cœur de la pratique enseignante. Toutefois, dans le cas du secondaire, le fait de ne pas pouvoir se contenter des contenus scientifiques acquis dans un premier cursus universitaire ne va pas de soi. Il est nécessaire de les compléter par une formation professionnalisante spécifique, axée sur la transposition didactique et la déconstruction-reconstruction de ces savoirs qu'il s'agit de transformer en savoirs à enseigner, auxquels s'ajoutent encore des savoirs pour enseigner propres aux sciences de l'éducation. Mais il n'en reste pas moins qu'au niveau des représentations des différents acteurs concernés, l'importance de cette phase de formation supplémentaire n'est de loin pas toujours reconnue : pourquoi faudrait-il en effet reprendre une formation alors que l'on vient d'en terminer une et qu'elle nous a en principe permis d'apprendre ce qui devait être transmis?

L'une des raisons de ces difficultés de perception réside peut-être dans un malentendu qui résulte de la séparation excessive et trop rigide qui éloigne la discipline de référence et sa didactique. En effet, une chercheuse l'a relevé à propos de la discipline histoire, mais ses propos peuvent sans doute valoir pour d'autres disciplines :

Le point d'ancrage sur lequel peuvent se lier et se développer de manière féconde et innovante la recherche disciplinaire et l'enseignement se situe moins sur les contenus scientifiques que sur les pratiques réflexives qui sont au cœur du renouvellement des sciences historiques et sociales et peuvent devenir le terreau d'un nouveau projet pour la formation des enseignants et l'enseignement de l'histoire. (Girault, 2015, p. 212)

La double évolution vers une professionnalisation des enseignants et une prise en compte des modalités de la transposition didactique au cœur des pratiques enseignantes rend dès lors pertinente, loin des barrières ou des asymétries traditionnellement instituées, une forme de dialogue et d'interaction réflexive entre les disciplines de référence du savoir scolaire et leurs didactiques. Mais cela implique alors que la formation des enseignants, en contact régulier avec la recherche, se démarque de tout enfermement dans le temps court des échéances immédiates de la pratique enseignante et ne se laisse pas réduire à sa seule dimension méthodologique.

Dans cette perspective, la formation des enseignants comprend des cours et séminaires qui apportent des éléments théoriques, des ateliers d'analyse des pratiques qui sont orientés soit sur une didactique 
disciplinaire, soit sur des éléments de sciences de l'éducation, ainsi qu'une pratique enseignante accompagnée, le plus souvent en pleine responsabilité pour le secondaire, mais qui est l'objet dans tous les cas d'un encadrement formatif fondé sur des observations et des analyses communes en collaboration avec des formateurs de terrain.

\section{L'articulation entre savoirs et pratique de terrain}

La nature de la relation avec la pratique de terrain constitue une originalité forte de la formation professionnalisante des enseignants genevois. Elle reste toutefois soumise à des tensions significatives. Dans le cas de l'école primaire, les étudiants sont accueillis dans les classes de formateurs de terrain pour des stages ponctuels ou filés dont l'organisation générale est actuellement en discussion, l'augmentation de la part de stages filés et de leur longueur permettant d'envisager davantage de continuité dans le cursus des étudiants. Pour le secondaire, la situation actuelle prévoit un stage en responsabilité à mi-temps sur une année, en principe la seconde, avec des stages en accompagnement complémentaires permettant aux étudiants d'être suffisamment au contact des deux niveaux d'enseignement, et en particulier du niveau gymnasial. Ce système est appelé à évoluer tout prochainement de manière significative dans le cadre d'une formation prévue en deux ans pour les deux niveaux d'enseignement et avec la possibilité de suivre la didactique de deux disciplines enseignables.

La formation universitaire des enseignants du secondaire telle qu'elle avait été conçue dans les années 2000 aurait pu être organisée sur la base de stages en accompagnement annuels. Mais cela aurait alors marqué une rupture peut-être trop forte avec une tradition genevoise de formation à l'enseignement en emploi salarié. Aujourd'hui, ce système a montré ses limites puisque, dans quelques disciplines, de nombreux étudiants restent bloqués dans leur cursus de formation faute d'obtenir un stage en responsabilité. Le nombre de ces stages dépend en effet étroitement du budget disponible pour les écoles, ce qui fait obstacle à une régulation même minimale du nombre d'étudiants. Le principe des stages en accompagnement annuels pourrait donc s'imposer à terme, au moins partiellement. Il faudrait alors qu'il soit conçu de manière à ce que l'étudiant puisse exercer petit à petit, et autant que possible, la pleine responsabilité de son enseignement. Mais cela permettrait en même temps de lever toute ambiguïté sur le caractère éminemment formateur de ce stage qui se distinguerait ainsi sans ambiguité d'un dispositif de formation en emploi.

Les conditions de reconnaissance de la formation des enseignants par la CDIP impliquent en effet que le principe d'alternance et d'interaction entre théories et pratique de terrain se donne à voir d'une manière crédible au cœur de l'organisation de la formation. L'application de ce principe ne va certes pas de soi. Mais il s'agit là clairement d'éviter un double écueil, celui d'une part d'une formation très théorique et trop détachée des réalités que vivent et quaffrontent les enseignants dans le quotidien de leurs classes; celui d'autre part d'une formation méthodologique immédiate, pour faire face aux problèmes les plus urgents dans les expériences de terrain, mais sans mise à distance réflexive ni apports susceptibles d'aider ces enseignants à construire une professionnalité qui puisse leur permettre de tenir dans la durée, de se diversifier et de rendre ainsi leur tâche potentiellement plus intéressante.

Dans cette perspective, la part du terrain et de l'expérience pratique dans la formation des enseignants ne s'oppose nullement à la dimension théorique et à l'accès à la recherche : l'une ne va pas sans l'autre. 
Mais il importe alors que les stages filés soient suffisamment longs pour permettre la réalisation de projets consistants, une ébauche de programmation et une prise de responsabilité aussi importante que possible.

\section{Les pressions vers le bas de l'espace politique}

$\mathrm{Au}$ moment d'écrire ces lignes, la formation des enseignants dans le contexte genevois est l'objet d'attaques politiques prégnantes dans l'espace public. Ainsi, pour la formation des enseignants du primaire, qui comprend un bachelor et un certificat complémentaire d'une année, soit 4 ans en tout ${ }^{4}$, une pression politique s'exerce pour une réduction à 3 ans, au niveau bachelor, apparemment conforme à ce qui se passe dans d'autres cantons. Mais cela aurait pour conséquence de priver les étudiants d'une première année d'enseignements dans le domaine des sciences de l'éducation; et surtout, cela ne pourrait pas permettre une formation complète qui prenne sérieusement en considération les différents secteurs de l'enseignement primaire. Quant à la formation dans le secondaire, elle est avant tout mise en discussion face aux nombreux étudiants de certaines disciplines qui sont restés bloqués en cours de route, pour certains depuis plusieurs années, étant donné l'absence de stages en responsabilité disponibles. Une réorganisation à venir des stages de cette formation devrait donc mener, notamment, à ce que la limitation du nombre d'étudiants s'effectue d'emblée, en amont, et non plus au milieu de la formation comme c'est le cas actuellement ${ }^{5}$.

Lélément le plus inquiétant qui émerge de cette situation concerne en fin de compte un déficit de reconnaissance, dans l'espace public et politique, de la nécessité pour les écoles et pour leurs élèves de cette formation des enseignants. Dans le fond, le métier d'enseignant du primaire ne serait pas un métier complexe, avec de gros défis à relever. Et le métier d'enseignant secondaire pourrait se contenter assez largement des apprentissages effectués dans le cadre de la formation universitaire disciplinaire. De telles représentations de sens commun, qui nont rien à voir avec ce qui se passe dans les classes, nuisent fortement à la formation initiale et continue des enseignants, mais surtout à la qualité des enseignements et apprentissages dans les écoles.

\section{Références}

Criblez, L. (2010). Die Reform der Lehrerinnen- und Lehrerbildung in der Schweiz seit 1990 : Reformprozesse, erste Bilanz une Desiderata. Dans H. Ambühl et W. Stadelmann (dir.), Tertiarisierung der Lehrerinnen- und Lehrerbildung (p. 22-58). Repéré à http://edudoc.ch/record/38225/files/StuB30A.pdf

Girault, B. (2015). De la didactique à l'épistémologie de l'histoire : une réflexivité partagée. Annales. Histoire, Sciences Sociales, 69(1), 205-214. http://dx.doi.org/10.1353/ahs.2015.0153

Schneuwly, B., Heimberg, C. et Villemin, R. (2012). Les didactiques au centre. Formation genevoise des enseignants du secondaire. Revue suisse des sciences de l'éducation, 34(3), 441-457. Repéré à http://rsse.elearninglab.org/wp-content/uploads/2013/06/SZBW 12.3 Schneuwly.pdf 


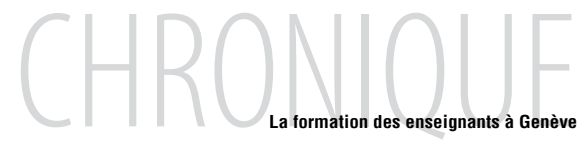

\section{Notes}

1 Avec, sur le plan fédéral, l'Accord intercantonal sur l'harmonisation de la scolarité obligatoire (concordat HarmoS) (http://www.edk.ch/dyn/11737.php).

2 En particulier, pour ce qui concerne la partie francophone du pays, un Plan d'études romand (PER) (https://www.plandetudes.ch/per).

3 L'enseignement secondaire II comprend des écoles de maturité (enseignement gymnasial), des écoles de diplôme ou des écoles professionnelles.

4 Voir les plans d'études actuels : $\underline{\text { http://www.unige.ch/iufe/enseignements/formations/enseignementprimaire.html }}$

5 Voir l'organisation actuelle de cette formation :

http://www.unige.ch/iufe/enseignements/formations/enseignementsecondaire.html 